\title{
New Vine Varieties for High Quality White Wine Production at RDSVV Bujoru
}

\author{
Alina DONICI*, Florin BORA, Aurel CIUBUCĂ, Viorica ENACHE, \\ Gabriel TABARANU \\ Research and Development Station for Vine and Winemaking Bujoru, Romania \\ *)Corresponding author, e-mail: donicialina79@gmail.com \\ BulletinUASVM Horticulture 73(2) / 2016 \\ Print ISSN 1843-5254, Electronic ISSN 1843-5394 \\ DOI:10.15835/buasvmcn-hort:12247
}

\begin{abstract}
The highest values for the absolute fertility coefficient (Cfa) and relative fertility coefficient (Cfr) were recorded for the 'Fetească regală' variety $(54.5 \pm 1.00 \%$ fertility; $1.60 \pm 1.00$ absolute coefficient (Cfa) and $0.86 \pm 1.00$ relative coefficient (Cfr). The results show that 'Bujoru' had the best values regarding the average weight of the grape bunch (303.5 $\pm 1.0 \mathrm{~g})$, weight of 100 berries $(278.07 \pm 1.0 \mathrm{~g})$, sugar content $(213.66 \pm 0.67 \mathrm{~g} / \mathrm{L})$ and average production ( $4.331 \pm 1.0 \mathrm{~kg} / \mathrm{vine})$. The new variety obtained, 'Bujoru', has a great potential to produce high quality white wines. Due to the large ammount of sugars that this variety accumulates, it can be successfully grown in other viticultural areas as well.
\end{abstract}

Keywords: grapevine, new variety, vineyard, wine

\section{Introduction}

In order to have a qualitative and quantitative wine production under the current climate change conditions, it is necessary to improve grapevine qualities by obtaining new cultivars. Climate conditions have a crucial role in the growth and development of grapevines (Fraga, 2013). Vine during its evolution has multiple known breeding methods, such as natural selection or directed (intraspecific, interspecific, clonal, genetic engineering, etc.). Nowadays it is a need to obtain new vine varieties that can provide both a high quality and high quantity production (Alexandrov, 2015 ).

\section{Aims and objectives}

The main aim of this research was to obtain new vine varieties with special characters and superior quality, based on the climate change phenomena from Romania.

\section{Materials and methods}

The grapevine varieties in this research are the following: 'Bujoru', 'Băbească gri' and 'Fetească regală'. In order to obtain the new variety of 'Bujo- ru' intraspecific hybridization method was applied using 'Băbească gri' and 'Pearl of Csaba' varieties. The new variety obtained, was approved in the year 2014 and the following fertility and productivity coefficients and indexes were determined: Cfa, Cfr, Ipa and Ipr.

\section{Results and Discussion}

Regarding the degree of fertility, the absolute fertility coefficient (Cfa) and the relative fertility coefficient ( $\mathrm{Cfr}$ ), it can be observed that the highest values were registered for the 'Fetească regală' variety $(54.5 \pm 1.00 \%$ fertility; $1.60 \pm 1.00$ - Cfa and $0.86 \pm 1.0$-Cfr). Regarding the absolute productivity index (Ipa) and the relative productivity index (Ipr) the highest values were observed in 'Bujoru'

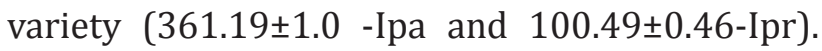
According to the physico-mechanical analysis the results show that 'Bujoru' varietyhad the best values for all the parameters analyzed $(303.5 \pm 1.0 \mathrm{~g}$ the average weight of the grape bunch, $278.07 \pm 1.0$ g weight of 100 berries; $213.66 \pm 0.67 \mathrm{~g} / \mathrm{L}$, sugar content and $4.331 \pm 1.0 \mathrm{~kg} /$ vine average production, except the 'Fetească regală' variety which had the 
Tab.1. The quality of different varieties at SCDVV Bujoru mean values for years 2013-2015 (mean \pm S.E. in $g$ )

\begin{tabular}{|c|c|c|c|c|c|}
\hline Cultivar & $\begin{array}{c}\text { Fertility } \\
\%\end{array}$ & $\begin{array}{l}\text { Absolute fertility } \\
\text { coefficient (Cfa) }\end{array}$ & $\begin{array}{l}\text { Relative fertility } \\
\text { coefficient (Cfr) }\end{array}$ & $\begin{array}{c}\text { Absolute } \\
\text { productivity } \\
\text { index } \\
\text { (Ipa) }\end{array}$ & $\begin{array}{c}\text { Relative } \\
\text { productivity } \\
\text { index } \\
\text { (Ipr) }\end{array}$ \\
\hline 'Bujoru' & $37.70 \pm 1.0 \mathrm{~b}$ & $1.19 \pm 0.429 b$ & $0.343 \pm 0.54 \mathrm{~b}$ & $361.19 \pm 1.0 \mathrm{a}$ & $100.49 \pm 0,46 \mathrm{a}$ \\
\hline 'Băbească gri' & $30.02 \pm 1.0 \mathrm{c}$ & $1.15 \pm 0.429 \mathrm{~b}$ & $0.316 \pm 0.54 \mathrm{~b}$ & $269.85 \pm 1.0 \mathrm{~b}$ & $77.71 \pm 0.156 \mathrm{~b}$ \\
\hline 'Fetească regală' & $54.50 \pm 1.0 \mathrm{a}$ & $1.60 \pm 1.0 \mathrm{a}$ & $0.860 \pm 1.00 \mathrm{a}$ & $167.91 \pm 1.0 \mathrm{c}$ & $92.08 \pm 0.156 \mathrm{ab}$ \\
\hline Average & $40.74 \pm 1.48$ & $1.316 \pm 0.002$ & $0.588 \pm 0.018$ & $266.3 \pm 4.0$ & $89.42 \pm 4.6$ \\
\hline $\begin{array}{l}\text { F (Fisher } \\
\text { Factor) }\end{array}$ & 23.783 & 33.245 & 99.615 & 194.44 & 2.456 \\
\hline Significance & $\mathrm{p} \leq \mathbf{0 . 0 0 0}$ & $\mathrm{p} \leq \mathbf{0 . 0 0 0}$ & $\mathrm{p} \leq \mathbf{0 . 0 0 0}$ & $\mathrm{p} \leq \mathbf{0 . 0 0 0}$ & $\mathrm{p} \leq 0.096$ \\
\hline
\end{tabular}

Tab.2. The quality of different varieties at SCDVV Bujoru mean values for years 2013-2015

\begin{tabular}{|c|c|c|c|c|c|}
\hline Cultivar & $\begin{array}{c}\text { Average weight } \\
\text { of a grape } \\
\text { bunch }(g)\end{array}$ & $\begin{array}{l}\text { Weight of } 100 \\
\text { berries }(\mathrm{g})\end{array}$ & $\begin{array}{c}\text { Must sugar } \\
\text { content (g/L) }\end{array}$ & $\begin{array}{c}\text { Total acidity } \\
\text { from must (g/ } \\
\left.\mathrm{H}_{2} \mathrm{SO}_{4}\right)\end{array}$ & $\begin{array}{c}\text { Average vine } \\
\text { production (kg/ } \\
\text { vine) }\end{array}$ \\
\hline 'Bujoru' & $303.50 \pm 1.0 \mathrm{a}$ & $278.07 \pm 1.0 \mathrm{a}$ & $213.66 \pm 0.67 \mathrm{a}$ & $4.050 \pm 1.0 \mathrm{~b}$ & $4.33 \pm 1.0 \mathrm{a}$ \\
\hline 'Băbească gri' & $233.50 \pm 1.0 \mathrm{~b}$ & $208.03 \pm 1.0 \mathrm{~b}$ & $215.30 \pm 0.67 \mathrm{a}$ & $4.50 \pm 0.13 \mathrm{a}$ & $2.97 \pm 0.43 \mathrm{~b}$ \\
\hline 'Fetească regală' & $106.40 \pm 1.0 \mathrm{c}$ & $167.21 \pm 1.0 \mathrm{c}$ & $203.00 \pm 1.0 \mathrm{~b}$ & $4.80 \pm 0.13 \mathrm{a}$ & $3.28 \pm 0.43 b$ \\
\hline Average & $214.47 \pm 2.63$ & $217.774 \pm 3.10$ & $210.66 \pm 1.59$ & $4.476 \pm 0.06$ & $3.531 \pm 0.16$ \\
\hline $\begin{array}{l}\text { F (Fisher } \\
\text { Factor) }\end{array}$ & 479.34 & 108.767 & 5.847 & 11.81 & 6.627 \\
\hline Significance & $\mathrm{p} \leq \mathbf{0 . 0 0 0}$ & $\mathrm{p} \leq \mathbf{0 . 0 0 0}$ & $\mathrm{p} \leq 0.005$ & $\mathrm{p} \leq \mathbf{0 . 0 0 0}$ & $\mathrm{p} \leq \mathbf{0 . 0 0 3}$ \\
\hline
\end{tabular}

Tab. 3. Analysis of the main quality parameters of the wine obtained at Dealu Bujorului Vineyard mean values for years 2013-2015

\begin{tabular}{cccccccc}
\hline Cultivar & $\begin{array}{c}\text { Alcohol } \\
(\% \text { vol. })\end{array}$ & $\begin{array}{c}\text { Total } \\
\text { acidity(g/L } \\
\text { C4H6O6) }\end{array}$ & $\begin{array}{c}\text { Volatile } \\
\text { acidity }(\mathrm{g} / \mathrm{L} \\
\mathrm{CH} 3 \mathrm{COOH})\end{array}$ & $\begin{array}{c}\text { Free SO2 } \\
(\mathrm{mg} / \mathrm{L})\end{array}$ & $\begin{array}{c}\text { Total SO2 } \\
(\mathrm{mg} / \mathrm{L})\end{array}$ & $\begin{array}{c}\text { Sugar } \\
(\mathrm{mg} / \mathrm{L})\end{array}$ & $\begin{array}{c}\text { Non-reducing } \\
\text { extract }(\mathrm{g} / \mathrm{L})\end{array}$ \\
\hline 'Bujoru' & $13.95 \pm 0.11 \mathrm{a}$ & $5.68 \pm 0.14 \mathrm{~b}$ & $0.35 \pm 1.0 \mathrm{~b}$ & $21.83 \pm 1.0 \mathrm{c}$ & $135 \pm 0.31 \mathrm{~b}$ & $1.16 \pm 1.0 \mathrm{c}$ & $24.16 \pm 0.2 \mathrm{~b}$ \\
\hline $\begin{array}{c}\text { 'Băbească } \\
\text { gri' }\end{array}$ & $12.46 \pm 1.0 \mathrm{~b}$ & $5.58 \pm 0.75 \mathrm{ab}$ & $0.46 \pm 1.0 \mathrm{a}$ & $28.5 \pm 1.0 \mathrm{~b}$ & $174 \pm 1.0 \mathrm{a}$ & $19.0 \pm 1.0 \mathrm{~b}$ & $23.5 \pm 0.2 \mathrm{~b}$ \\
\hline $\begin{array}{c}\text { 'Fetească } \\
\text { regală' }\end{array}$ & $14.5 \pm 0.11 \mathrm{a}$ & $6.35 \pm 0.75 \mathrm{a}$ & $0.29 \pm 1.0 \mathrm{c}$ & $33.9 \pm 1.0 \mathrm{a}$ & $130 \pm 0.31 \mathrm{~b}$ & $26.5 \pm 1.0 \mathrm{a}$ & $26.6 \pm 1.0 \mathrm{a}$ \\
\hline $\begin{array}{c}\text { Average } \\
\mathbf{1 3 . 6 4 \pm 0 . 1 4}\end{array}$ & $\mathbf{6 . 0 7} \pm \mathbf{0 . 0 8}$ & $\mathbf{0 . 3 7 2 \pm 0 . 0 1}$ & $\mathbf{2 8 . 0 7 8} \pm \mathbf{0 . 8 3}$ & $\mathbf{1 4 6 . 3 3} \pm \mathbf{1 . 9 9}$ & $\mathbf{1 5 . 5 7 \pm 1 . 0 3}$ & $\mathbf{2 4 . 7 5 6 \pm 0 . 2 4}$ \\
\hline $\begin{array}{c}\text { F (Fisher } \\
\text { Factor) }\end{array}$ & $\mathbf{1 7 . 6 2 5}$ & $\mathbf{5 . 4 0 1}$ & $\mathbf{3 0 . 6 3 4}$ & $\mathbf{1 7 . 6 9 3}$ & $\mathbf{4 8 . 7 7 3}$ & $\mathbf{5 3 . 7 2 2}$ & $\mathbf{1 5 . 0 5 8}$ \\
\hline Significance & $\mathbf{p} \leq \mathbf{0 . 0 0 0}$ & $\mathbf{p} \leq \mathbf{0 . 0 0 7}$ & $\mathbf{p} \leq \mathbf{0 . 0 0 0}$ & $\mathbf{p} \leq \mathbf{0 . 0 0 0}$ & $\mathbf{p} \leq \mathbf{0 . 0 0 0}$ & $\mathbf{p} \leq \mathbf{0 . 0 0 0}$ & $\mathbf{p} \leq \mathbf{0 . 0 0 0}$ \\
\hline
\end{tabular}


highest value in terms of total acidity: $4.8 \pm 0.13$ (g/L $\mathrm{H}_{2} \mathrm{SO}_{4}$ (Tab.2).In Tab. 3 the technological potential of the three vine varieties analysed in this study are shown. The average value of the alcohol content during the three years of study, clearly show a sensitivity of 'Bujoru' variety to climatic conditions in the south-east of the country in terms of biosynthesis of carbohydrates. The average value of wine alcohol content was $13.95 \pm 0.11 \%$ vol. The acidity registered at the 'Bujoru' cultivar was $5.68 \pm 0.14 \mathrm{~g} / \mathrm{L} \mathrm{C}_{4} \mathrm{H}_{6} \mathrm{O}_{6}$. Non-reducing extract is an important parameter for wine, and values noted are statistically equal, in terms of the variety 'Bujoru' the registered value was $24.16 \pm 0.2 \mathrm{~g} / \mathrm{L}$ compared to the value recorded from the variety 'Babeasca gri' 23.5 $\pm 0.2 \mathrm{~g} / \mathrm{L}$.

\section{Conclusion}

The new variety 'Bujoru' has a great potential to produce high quality white wines. Due to the large amount of sugars that accumulates this new cultivar can be successfully cultivated in different viticultural areas from Romania.

\section{REFERENCES:}

1. Alexandrov E (2015). New requirements to create of vine varieties economic and environmental significance. J UASMV Cluj Agriculture Science and Practice 3-4:44-51

2. Fraga H, Malheiro AC, Moutinho-Pereira J, Santos JA, (2013). Future scenarios for viticultural zoning in Europe: ensemble projections and uncertainties. Int J Biometeorol 57(6):909-25. 